\title{
Variable Selection Using Shrinkage Priors
}

\author{
Hanning $\mathrm{Li}^{*}$, Debdeep Pati \\ Department of Statistics, Florida State University, Tallahassee, FL 32306
}

\begin{abstract}
Variable selection has received widespread attention over the last decade as we routinely encounter high-throughput datasets in complex biological and environment research. Most Bayesian variable selection methods are restricted to mixture priors having separate components for characterizing the signal and the noise. However, such priors encounter computational issues in high dimensions. This has motivated continuous shrinkage priors, resembling the two-component priors facilitating computation and interpretability. While such priors are widely used for estimating high-dimensional sparse vectors, selecting a subset of variables remains a daunting task. A general approach for variable selection with shrinkage priors is proposed. The presence of very few tuning parameters makes our method attractive in comparison to adhoc thresholding approaches. The applicability of the approach is not limited to continuous shrinkage priors, but can be used along with any shrinkage prior. Theoretical properties for near-collinear design matrices are investigated and the method is shown to have good performance in a wide range of synthetic data examples and in a real data example on selecting genes affecting survival due to lymphoma.
\end{abstract}

Keywords: Bayesian, Horseshoe, Markov Chain Monte Carlo, Shrinkage priors, Variable selection

\section{Introduction}

Variable selection in high-dimensional models has received substantial interest in recent years [1] and is a challenging problem for Bayesians. With rapid ad-

\footnotetext{
*Corresponding author: 214 Rogers Building, 117 North Woodward Avenue, P.O. Box 3064330, Tallahassee, FL 32306-4330. Ph: (850) 644-3218, Fax: (850) 644-5271

Email addresses: h. Ii@stat.fsu . edu (Hanning Li), debdeep@stat. fsu .edu (Debdeep Pati)
} 
vances in data acquisition and storage techniques, modern scientific investigation$\mathrm{s}$ in epidemiology, genomics, imaging and networks are increasingly producing more variables compared to the sample size. One hope for meaningful inferences in such situations is to discover a subset of variables that explains the physical or biological process generating the data. Exploiting such underlying structures, commonly prevalent in the form of sparsity of model parameters, holds the key to meaningful inferences in high-dimensional settings.

This article revisits the problem of Bayesian variable selection in the context of Gaussian linear models (1) using shrinkage priors:

$$
Y=X \beta+\epsilon, \quad \epsilon \sim N\left(0, \sigma^{2} I_{n}\right)
$$

where $Y$ is an $n$-dimensional response observed with respect to the $n \times p$ covariate matrix $X$ and $\beta$ is the $p$-dimensional coefficient vector. Traditionally, to select the important variables out of $X_{1}, \ldots, X_{p}$, a two component mixture prior (also referred to as a spike-and-slab prior) $[2,3,4]$ is placed on $\beta$. These priors include a mass or a spike at zero characterizing the noise and a continuous density (usually centered at zero) representing the signal density.

Although these priors are highly appealing in allowing separate control of the level of sparsity and the size of the signal coefficients, they lead to computational hurdles in high-dimensions due to the need to explore a $2^{p}$ model space. [5] recently showed a startling selection inconsistency phenomenon for several commonly used spike-and-slab priors based on intrinsic Bayes factors [6], fractional Bayes factors [7], and g-priors [8] when the model size $p \gtrsim \sqrt{n}$. This behavior was attributed to the common practice of centering the prior on the signal component at zero (local prior), which obliterates the demarcation between the signal and noise in high dimensions and leads to negligible posterior probability being assigned to any given model. [5] advocated the use of non-local priors to obtain selection consistency when $p=O(n)$, where the density for the signals decays to zero in a neighborhood of the origin. When $p \gg n$, it is not immediately clear whether non-local prior distributions can provide sufficient distinguishability between the signals and the noise coefficients.

Nevertheless, the practical problem of selecting variables has been a major bottleneck even with spike-and-slab priors. Although the highest posterior probability model (HPPM) is commonly perceived as the best model [9, 10], it is not optimal for prediction [11] since HPPM is the Bayes estimate only under 0-1 loss function. Moreover, finding HPPM in high-dimensions is computationally demanding since the Markov chain Monte Carlo (MCMC) samples can only visit a 
minute fraction of the $2^{p}$ model space even for a relatively large number of Gibb$\mathrm{s}$ iterations. To circumvent these issues, [11] proposed the median probability model (MPM) defined as the model consisting of those variables which have an overall posterior probability of inclusion greater than or equal to $1 / 2$. Although this is the optimal predictive model, [12] found that summaries of the posterior distribution based on marginal and joint distributions may give conflicting results for assessing the importance of strongly correlated covariates.

Computational issues and considerations that many of the $\beta_{j}$ s may be small but not exactly zero have led to a rich variety of continuous shrinkage priors being proposed recently $[13,14,15,16,17,18]$, which can be unified through a globallocal (GL) scale mixture representation of [19] below,

$$
\beta_{j} \sim \mathrm{N}\left(0, \lambda_{j}^{2} \tau^{2}\right), \quad \tau \sim f, \quad \lambda_{j} \sim g
$$

where $f$ and $g$ are densities on the positive real line. In (2), $\tau$ controls global shrinkage towards the origin while the local parameters $\lambda_{j} \mathrm{~s}$ allow deviations in the degree of shrinkage. Special cases include Bayesian Lasso [13], relevance vector machine [14], normal-gamma mixtures [15] and the horseshoe $[16,17]$ among others. GL priors potentially have substantial computational advantages over mixture priors, since the normal scale mixture representation allows for conjugate updating of $\beta$ and $\lambda$ in blocks. Moreover, a number of frequentist regularization procedures such as ridge, Lasso, bridge and elastic net correspond to posterior modes under GL priors with appropriate choices of $f$ and $g$.

The literature on model selection with continuous shrinkage priors is even less-developed due to the unavailability of exact zeros in the posterior samples of $\beta$. Heuristic methods based on thresholding the posterior mean $/$ median of $\beta$ are often used in practice which lack theoretical justification, and inference is highly sensitive to the choice of the threshold. There is a recent literature on decoupling shrinkage and selection [20,21,22], which poses the problem of selection as a loss function based decision rather than inducing sparsity through a prior distribution. A naive way to select variables using a shrinkage prior is to see whether the posterior credible interval contains zero or not. Such a method usually has a poor performance because it is very difficult to estimate the uncertainty accurately in high dimensional problems.

In this article, we aim to address the problem of selecting variables through a novel method of post processing the posterior samples. The approach is based on first obtaining a posterior distribution of the number of signals by clustering the signal and the noise coefficients and then estimating the signals from the posterior 
median. This simple approach requires very few tuning parameters and is shown to have excellent performance relative to both existing frequentist and Bayesian approaches. Moreover, the method is not only applicable to continuous shrinkage priors, but also can be used along with any shrinkage prior for $\beta$ after a full M$\mathrm{CMC}$ run. For the ease of exposition, we focus on the spike-and-slab prior and the horseshoe prior and compare the performances using HPPM, MPM and the credible set approach for variable selection. Interestingly, in the presence of high collinearity among the covariates, we demonstrated better performance when the horseshoe prior is used in conjunction with our selection procedure.

The organization of the present paper is as follows. Section 2 describes the variable selection algorithm. Theoretical properties for collinear design matrices are considered in Section 3. Section 4 contains detailed comparisons in synthetic data. A real data application on selecting important genes for predicting survival from lymphoma is illustrated in Section 5. A discussion is provided in Section 6.

\section{Methodology}

Our objective is to develop an algorithm to select the important variables based on the posterior samples of $\beta$ obtained from the MCMC samples in (1) when a shrinkage prior is placed on $\beta$. The algorithm is independent of the prior for $\beta$, but dependent on the linear model with additive error assumption in (1). Unlike existing approaches, the method involves very few tuning parameters, hence readily suitable for future use of practitioners. Our idea is based on finding the most probable set of variables in the posterior median of $\beta$. Since the distribution of the number of important variables is more stable and is largely unaffected by the mixing of the MCMC, we propose to first find the mode $H$ of the distribution of number of important variables and then select the $H$ largest coefficients from the posterior median of $\left|\beta_{j}\right|$.

\subsection{2-means (2-M) variable selection}

We expect two clusters of $\left|\beta_{j}\right|$, with one concentrated closely near zero corresponding to noise variables and the other one away from zero corresponding to the signals. As an automated approach, we cluster $\left|\beta_{j}\right| \mathrm{s}$ at each MCMC iteration using $k$-means with $k=2$ clusters. For the $i$ th iteration, the number of non-zero signals $h_{i}$ is then estimated by the smaller cluster size out of the two clusters. A final estimate $(H)$ of the number of non-zero signals is obtained by taking the mode over all the MCMC iterations, i.e., $H=\operatorname{mode}\left\{h_{i}\right\}$. The $H$ largest entries of the posterior median of $|\beta|$ are identified as the non-zero signals. 
When the true coefficient vector $\beta_{T}$ has signal coefficients varying in the signal strengths, 2-M variable selection approach described above may inappropriately cluster the smaller signals together with the noise variables. A possible solution is to use different values of the number of clusters $k$, but it is usually difficult and time-consuming to find the optimal value of $k$. To deal with this, we propose a simple modification called the sequential 2-means below.

\subsection{Sequential 2-means $\left(S_{2} M\right)$ variable selection}

\subsubsection{Algorithm:}

We start with a few notations. Define $S_{T}$ to be the indices of non-zero signals in $\beta_{T}$ and $S_{E}$ to be the indices of the selected covariates. To assess the efficacy of a variable selection procedure, we introduce two types of errors a) $\left|S_{T} \cap S_{E}^{c}\right|$ : masking error (also called 'false negatives'), and b) $\left|S_{E} \cap S_{T}^{c}\right|$ : swamping error (also called 'false positives').

When $\beta_{T}$ has different levels of signal strengths, the 2-M variable selection approach will have a high chance of incurring masking error. In other words, it is highly likely that some true signals with low signal strengths will be clustered with the noise coefficients even when the corresponding $\beta_{j}$ s are estimated well.

Our main motivation to propose the sequential 2-means $\left(\mathrm{S}_{2} \mathrm{M}\right)$ variable selection approach is to reduce the probability of masking error. Let $b>0$ be a tuning parameter, then $\mathrm{S}_{2} \mathrm{M}$ is defined as below. On the $i$ th MCMC sample of $\beta$ :

I perform a 2-means algorithm on $\left|\beta_{j}\right|, j=1,2, \ldots, p$. Denote the cluster means with smaller values by $m$ and the one with large values by $M$ ( $m \leq$ $M)$. Initialize a set $D$ with all the indices from the cluster with the smaller mean $m$. While the difference $M-m$ is greater than $b$ :

(a) update $D$ to be all the indices from the cluster with the smaller mean $m$;

(b) perform a 2-means on $\left|\beta_{j}\right|, j \in D$;

(c) update $m$ and $M$ to be two cluster means ( $m \leq M$ ) obtained from (b).

II The set $D$ is considered to contain coefficients of noise covariates. So the estimated number of signals $h_{i}$ is $p-|D|$.

The above algorithm is repeated for all MCMC samples of $\beta$ and the final estimate of the number of signals $H$ as well as the variable selection are obtained in the same way as in the 2-M algorithm. 


\subsubsection{Choosing tuning parameter $b$ :}

Using an appropriate tuning parameter $b, \mathrm{~S}_{2} \mathrm{M}$ is capable of reducing the chance of masking error. However, note that this occurs at the cost of an increased probability of swamping error. A larger value of $b$ tends to increase the masking error, while a smaller value of $b$ increases swamping error. For sufficiently large $b, \mathrm{~S}_{2} \mathrm{M}$ essentially reduces to $2-\mathrm{M}$.

Hence one should choose $b$ so that the sum of the two errors is minimized. In order to assess the important factors influencing the choice of $b$, let us first consider a noise-free version of the model in (1) and hypothesize the ideal situation when the Bayesian procedure provides exactly accurate estimation of $\beta_{T}$ at every iteration. Then it is not hard to conclude any value of $b$ between 0 and the lowest absolute signal strength leads to correct variable selection. However, in presence of noise, it is impossible for any method to produce an exact estimate of $\beta_{T}$. In addition, using continuous shrinkage priors, the estimated coefficients for the noise variables will never be exactly zero. As the noise level $\sigma$ increases, the estimates for the coefficients become more variable. This makes it more likely for the nonzero coefficients of lower signal strength to be clustered with the noise coefficients leading to an increase in the masking error. Hence we believe a proper value for $b$ should be somehow positively correlated the posterior estimate of $\sigma$. The estimate of $\sigma$ in (1) is affected by i) the true noise level ii) collinearity in the design matrix iii) as well as the ill-posed-ness of the high-dimensional regression problem i.e., how large $p$ is compared to $n$. These key factors contribute to the accuracy of the selection procedure. However, it is well-known that the variance $\sigma^{2}$ is usually difficult to estimate accurately especially in high-dimensional settings.

In practice, we propose the following procedure to determine the tuning parameter $b$. First, we start with a conservative interval $[L, U]$ and construct an equally separated points $\left\{b_{i}\right\}$ inside $[L, U]$. Then we run the $\mathrm{S}_{2} \mathrm{M}$ algorithm for each $b_{i}$ and denote the estimated number of signals by $H_{b_{i}}$. The tuning parameter $b$ (or equivalently the estimated number of signals $H$ ) is determined by looking at the plot $\left\{b_{i}\right\}$ v.s. $\left\{H_{b_{i}}\right\}$ following the visual criteria: identify a sharp decrease associated with the highest static level for $H_{b_{i}}$. As discussed before, a very small value of $b$ increases the swamping error. Thus, a sharp decrease in $H_{b_{i}}$ in this plot indicates that the swamping error has been significantly reduced for the corresponding $b_{i}$. Out of several sharp decreases, the one that is followed by the highest static level for $H_{b_{i}}$ is associated with the minimum masking error. Section 4.2 later illustrates this process in details through two simulation examples. The entire procedure for variable selection is post-MCMC; hence obtaining $b_{i}$-v.s.- $H_{b_{i}}$ 
is computationally efficient.

\section{Dealing with correlated predictors using continuous shrinkage priors}

In presence of confounders which are highly correlated with an important predictor, it is crucial that a variable selection method can identify the true predictor. $[18,23]$ recently showed that a global-local shrinkage prior (2) achieves better concentration around sparse vectors in comparison with shrinkage prior based on only global scale i.e., setting $\lambda_{j} \equiv 1$ in (2). In this section, we show that such observations extend to the case of variable selection. While a "global-only" shrinkage prior fails to select the true variables under moderate correlation, an appropriately constructed global-local shrinkage prior can achieve desirable variable selection even under high correlation.

For the ease of understanding the behavior of continuous shrinkage priors under correlation, consider only two covariates where variable 1 is the important predictor and variable 2 is the confounder.

Let $X^{\prime} X=[1 \rho ; \rho 1]$ with $\rho$ characterizing the correlation between the two predictors. Assume $\hat{\beta}_{M L E, j}$ and $\hat{\beta}_{S, j}$ are the maximum likelihood estimate and posterior mean of $\beta_{j}, j=1,2$ respectively. We empirically observe that MLE underestimates the signal coefficient $\beta_{1}$ and over-estimates the confounder $\beta_{2}$ under high correlation. Ideally, a shrinkage prior should counter-balance this effect allowing the corresponding posterior estimates to be well-separated, thus facilitating variable selection. We define a terminology called reverse-shrinkage to describe this. A prior is said to satisfy reverse-shrinkage if $\left|\hat{\beta}_{M L E, 1}\right| \geq\left|\hat{\beta}_{M L E, 2}\right|$ implies $\left|\hat{\beta}_{S, 1} / \hat{\beta}_{S, 2}\right| \geq\left|\hat{\beta}_{M L E, 1} / \hat{\beta}_{M L E, 2}\right|$. Suppose we can write a Bayes estimator under a shrinkage prior as a function of the MLE: $\hat{\beta}_{S, j}=\left[1-S_{j}\left(\hat{\beta}_{M L E}\right)\right] \hat{\beta}_{M L E, j}$, $j=1,2, \ldots, p$, where the shrinkage $S_{j}$ is less than 1 . Then reverse-shrinkage means a larger magnitude of MLE results in a smaller shrinkage. Clearly, this is a desirable phenomenon for any variable selection approach.

Theorem 3.1. Suppose $\beta_{i} \sim N\left(0, \sigma^{2} \tau^{2}\right), i=1,2$, with $\sigma^{2}$ defined in (1), and the $n \times 2$ covariate $X$ satisfies $X^{\prime} X=[1 \rho ; \rho 1]$ where $\rho \in(0,1)$. Then if $\left|\beta_{M L E, 1}\right|>\left|\beta_{M L E, 2}\right|$,

$$
\left|\frac{\hat{\beta}_{N, 1}}{\hat{\beta}_{N, 2}}\right|<\left|\frac{\hat{\beta}_{M L E, 1}}{\hat{\beta}_{M L E, 2}}\right|,
$$

for any $\tau$ and $n>2$, where $\hat{\beta}_{N}$ denotes the posterior mean. 
Hence, when for correlated predictors, shrinkage priors with only global shrinkage parameters are no better than using MLEs. The proof of Theorem 3.1 is provided in Appendix A.

Next, we turn our attention to global-local shrinkage priors. We focus on the horseshoe prior for a fixed value of the global shrinkage parameter $\tau$

$$
\beta_{j} \mid \lambda_{j}, \tau \sim N\left(0, \lambda_{j}^{2} \tau^{2}\right), \quad \lambda_{j} \sim \sqrt{\mathrm{Ca}^{+}(0,1)},
$$

where $\mathrm{Ca}^{+}(0,1)$ denotes the standard half-Cauchy distribution with pdf $2 /\{\pi(1+$ $\left.\left.x^{2}\right)\right\}$ for $x>0$.

With $X^{\prime} X$ defined in Theorem 3.1, we write the HS estimators as functions of MLEs in Lemma Appendix A.4. More precisely, the HS estimators are functions of $\rho, \tau, \hat{\beta}_{M L E, 2}$, and $A=\left|\hat{\beta}_{M L E, 1} / \hat{\beta}_{M L E, 2}\right|$. Given values for these parameters, we calculate the approximate values of $\left|\hat{\beta}_{H S, 1} / \hat{\beta}_{H S, 2}\right|$ and $\left|\hat{\beta}_{M L E, 1} / \hat{\beta}_{M L E, 2}\right|$ in Matlab to see whether reverse-shrinkage occurs.

Through the following figure, we will show the horseshoe prior can be made to satisfy the reverse-shrinkage property by suitably choosing $\tau$. Figure 1 provides numerical analysis with $\hat{\beta}_{M L E, 2}=1$ and 1.5 , for different values of $\rho \in$ [0.94, 0.99], $\tau \in(0,1)$, and $A>1$. Blue / red dots indicate reverse-shrinkage / lack of it. Figure 1 shows reverse-shrinkage is more likely to occur when there are a smaller value of $\rho$, a greater value of $A$ (these two observations are expected) and a smaller value of $\tau$ present. The $\rho$ and $A$ are directly dependent on data, however, by choosing $\tau$ carefully, it is possible to increase the possibility of achieving reverse-shrinkage. Clearly, for values of $\rho$ close to 1 , the horseshoe prior with large values of $\tau$ is less prone to achieve the reverse-shrinkage compared to smaller values of $\tau$. [16,17] have pointed out $\tau$ is supposed to be estimated small and have a substantial mass around zero in order to efficiently capture the sparsity in $\beta$. In practice, we suggest to use an upper bound for the global hyperparameter $\tau$.

\section{Simulation Study}

In this section we compare the performance of the methods proposed in this article, i.e. $\mathrm{S}_{2} \mathrm{M}$ and 2-M, with other competing methods in terms of variable selection, especially when there is high collinearity present among the covariates. We consider the horseshoe (HS), the spike-and-slab (SS) and the adaptive Lasso 


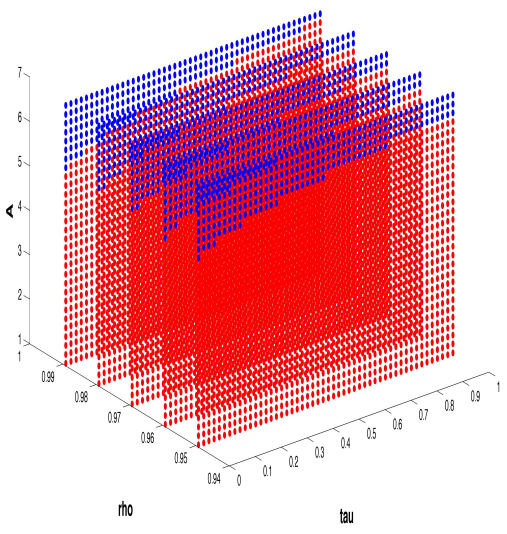

(a) $\hat{\beta}_{M L E, 2}=1$

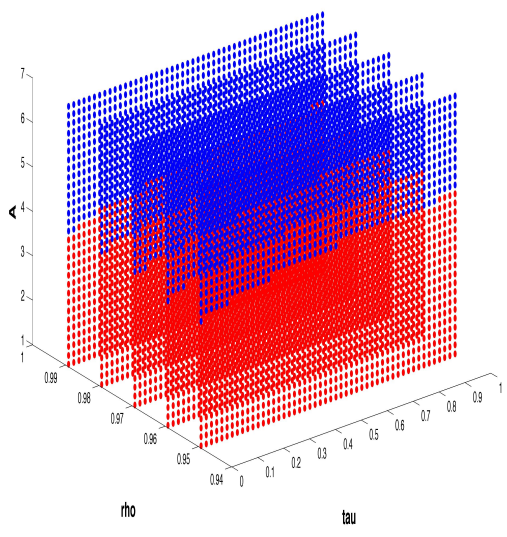

(b) $\hat{\beta}_{M L E, 2}=1.5$

Figure 1: +ve reverse-shrinkage (blue), -ve reverse-shrinkage (red) for various $A, \rho$ and $\tau$

(AL) [24]. To implement SS, we assume

$$
\begin{gathered}
\beta_{j} \mid \pi, \omega_{j}^{2}, \sigma \sim \pi \delta_{0}+(1-\pi) N\left(0, \sigma^{2} \omega_{j}^{2}\right), \\
\omega_{j}^{2} \sim \operatorname{IG}(3 / 2,3 / 2), 1-\pi \sim \operatorname{Beta}(1,15), \sigma^{2} \sim \operatorname{IG}(3 / 2,3 / 2) .
\end{gathered}
$$

The HS is used as in [16]:

$$
\begin{gathered}
\beta_{j} \mid \lambda_{j}, \tau, \sigma^{2} \sim \mathrm{N}\left(0, \lambda_{j}^{2} \tau^{2} \sigma^{2}\right), \\
\lambda_{j} \sim \sqrt{\mathrm{Ca}^{+}(0,1)}, \tau \sim \sqrt{\mathrm{Ca}^{+}(0,1)}, \sigma^{2} \sim \mathrm{IG}(3 / 2,3 / 2) .
\end{gathered}
$$

$[16,17]$ suggested thresholding posterior estimates of $\kappa_{j}=1 /\left(1+\lambda_{j}\right)$ at $1 / 2$ to perform variable selection in HS. We refer to this as the Hypothesis Testing approach, abbreviated as HT. Although a suitable $\kappa_{j}$ can be defined in the GL family (2), we found HT to be most effective with HS. It is important to reiterate here that the algorithm $\mathrm{S}_{2} \mathrm{M}$ is independent of the prior for $\beta$. We also used the credible set approach, abbreviated as CS. To implement AL, we used the parcor package in R. For HS, the variable selection approaches tried are: $\mathrm{S}_{2} \mathrm{M}, 2-\mathrm{M}, \mathrm{CS}$ and HT, while for SS we tried $\mathrm{S}_{2} \mathrm{M}, 2-\mathrm{M}, \mathrm{HPPM}$ and MPM. The error variance when generating $Y$ in (1) was 1 in every simulation study in this section. For the Bayesian procedures, the MCMC was run for 5,000 iterations discarding a burn- 
in of 2,000. Convergence was monitored using standard tests and diagnostic trace plots (refer to Appendix B).

\subsection{Simulation examples: set 1}

We start with four combinations of sample size $(n)$, the number of covariates $(p)$, the number of signals $(r)$ and signal strength (magnitude of the signal, denoted by $B$ ). (1) $n=50, p=300, r=10, B=2$; (2) $n=50, p=300, r=10, B=4$; (3) $n=100, p=800, r=20, B=2$ and (4) $n=100, p=800, r=20, B=4$. Under each setting, we considered an "uncorrelated covariate" setting (uncor) and a "correlated covariate" setting ( cor $)$. Covariates are generated from standard normal distributions independently and an intercept is included. The covariate matrix corresponding to cor contains two pairs of correlated covariates, and in each pair, one is a signal while the other is a noise predictor. Both correlations are above 0.99 .

Results presented in Table 1 are obtained by averaging over 20 replicates of observations obtained using the same covariate matrix. The first number in each parenthesis in Table 1 is the masking error and the second number is the swamping error. The tuning parameter $b$ in $S_{2} \mathrm{M}$ is set to be $2 \hat{\sigma}^{2}$ (refer to Section 4.2).

In general, HS out-performs SS and AL significantly. We found the performance of our proposed method, $\mathrm{S}_{2} \mathrm{M}$ (or 2-M) to be consistently better when used in conjunction with HS, even when high collinearity is present between the covariates. CS often selects the wrong one between a highly correlated pair of covariates. When the difficulty of the variable selection task is high, (refer to columns 2, 4, 6 and 8 in Table 1), CS masked a larger fraction of the true signals. The performance of HS+HT is better than CS, but worse than $\mathrm{S}_{2} \mathrm{M}$ (or 2-M) (refer to columns 2 and 6 in Table 1). In addition, we have to note here HT is applicable to priors from only the GL family, while our proposed methods are applicable to any shrinkage priors.

SS fails to estimate accurately when $p$ is large (refer to the right half of Table 1). In this case, $S_{2} M$ (or 2-M) resulted in higher swamping errors but much lower masking errors compared to HPPM and MPM. $\mathrm{S}_{2} \mathrm{M}+\mathrm{HS}$ out-performs AL.

\subsection{Simulation examples: set 2}

In this example, we compare $\mathrm{S}_{2} \mathrm{M}$ and 2-M, when the true coefficient vector $\beta_{T}$ contains different levels of signal strengths. We conduct this to illustrate the approach proposed in Section 2.2 for choosing the value of $b$.

We set $n=50$ with $p=300$ and $r=10$. The true coefficient vector $\beta_{T}$ is set to be $\beta_{T_{1}}=\left\{6,(3)_{4},(1)_{5},(0)_{290}\right\}$, where $(x)_{k}$ denotes a vector formed by 
Table 1: Masking and Swamping errors

\begin{tabular}{ccccccccc}
\hline $\begin{array}{c}n \\
n\end{array}, p, r$ & \multicolumn{3}{c}{$n=50, p=300, r=10$} & \multicolumn{3}{c}{$n=100, p=800, r=20$} \\
$B$ & 2 & 2 & 4 & 4 & 2 & 2 & 4 & 4 \\
& uncor & cor & uncor & cor & uncor & cor & uncor & cor \\
\hline $\mathrm{HS}+\mathrm{S}_{2} \mathrm{M}$ & $(0,0)$ & $(0.5,0.5)$ & $(0,0)$ & $(0.3,0.3)$ & $(0.7,0.75)$ & $(0.4,0.4)$ & $(0,0)$ & $(0.15,0.15)$ \\
$\mathrm{HS}+2 \mathrm{M}$ & $(0,0)$ & $(0.5,0.5)$ & $(0,0)$ & $(0.3,0.3)$ & $(0.7,0.75)$ & $(0.4,0.4)$ & $(0,0)$ & $(0.15,0.15)$ \\
$\mathrm{HS}+\mathrm{CS}$ & $(0,0)$ & $(2.2,0)$ & $(0,0)$ & $(1.7,0)$ & $(2.65,0.0)$ & $(2.65,0.05)$ & $(1.35,0)$ & $(1,0)$ \\
$\mathrm{HS}+\mathrm{HT}$ & $(0,0)$ & $(1.7,0.25)$ & $(0,0)$ & $(0.4,0.2)$ & $(1.75,0.0)$ & $(1.3,0.1)$ & $(0,0)$ & $(0.2,0.05)$ \\
\hline $\mathrm{SS}+\mathrm{S}_{2} \mathrm{M}$ & $(0,0)$ & $(0.75,0.75)$ & $(0,0)$ & $(0.35,0.35)$ & $(0,16.6)$ & $(0.2,17.75)$ & $(0.15,41.4)$ & $(0.2,23.35)$ \\
$\mathrm{SS}+2 \mathrm{M}$ & $(0,0)$ & $(0.75,0.75)$ & $(0,0)$ & $(0.35,0.35)$ & $(0,17.2)$ & $(0.2,17.4)$ & $(0.15,42.05)$ & $(0.2,23.8)$ \\
$\mathrm{SS}+\mathrm{HPPM}$ & $(0,0)$ & $(0.75,0.75)$ & $(0,0)$ & $(0.35,0.35)$ & $(8.9,3.5)$ & $(11,4.7)$ & $(13.9,5.25)$ & $(9.95,4.2)$ \\
$\mathrm{SS}+\mathrm{MPM}$ & $(0,0)$ & $(0.45,0.95)$ & $(0,0)$ & $(0.20,0.55)$ & $(6.7,1.4)$ & $(6.55,1.6)$ & $(9.45,4.2)$ & $(7.6,2.45)$ \\
\hline $\mathrm{AL}$ & $(0,1.4)$ & $(1,2,3.2)$ & $(0,0.35)$ & $(0.8,1.4)$ & $(6.55,1.5)$ & $(7.7,4)$ & $(0.10,0.15)$ & $(1.8,3.15)$ \\
\hline
\end{tabular}

replicating $x$ for $k$ times. There are two noise covariates, which are correlated to one signal of strength 3 and one signal of strength 1 respectively. Both correlations are above 0.99 .

Post-MCMC, we create a sequence $b_{i}$ of length 20, which equally divide the interval $\left(0.5 \hat{\sigma}^{2}, 10 \hat{\sigma}^{2}\right)$. For each $b_{i}$, we perform $\mathrm{S}_{2} \mathrm{M}$ and record $H_{b_{i}}$ as well as the corresponding set of selected signals. The left panel in Figure 2 shows the $b_{i}$-v.s.$H_{b_{i}}$ plot for $n=50, p=300, r=10, \beta_{T}=\beta_{T 1}$. When $b$ is close to zero, $H_{b}$ is as large as 51. This is expected since a very small value of $b$ leads to large swamping error. Moreover, $H_{b_{i}}$ decreases sharply from a large value and remains static at 10 when $b_{i}$ is between 0.11 and 0.88 . When $b_{i}$ further increases approaching the lowest signal strength, $H_{b_{i}}$ decreases to and remains at 5 , which indicates the five weaker signals are masked as noise variables. The plot indicates the estimated number of signals $(H)$ to be 10 instead of 5 . The pairs of errors using $\mathrm{S}_{2} \mathrm{M}$, 2M, CS, HT are $(0,0),(5,0),(1,0)$, and $(5,0)$ respectively. The 2-M masked the 5 weaker signals and $\mathrm{S}_{2} \mathrm{M}$ resulted in smaller errors compared with either CS or HT mainly due to its ability to deal with correlated covariates.

In the right panel of Figure 2, we display the $b_{i}$-V.s. $-H_{b_{i}}$ plot for $n=50, p=$ $200, r=10$, with the true coefficient vector $\beta_{T}=\beta_{T 2}=\left\{4,(2)_{4},(1)_{5},(0)_{190}\right\}$ and two pairs of correlated covariates (one signal correlated with one noise variable) with correlations above 0.99 . As before, we concluded this plot indicated $H$ to be 10 instead of 5 . The pairs of errors using $\mathrm{S}_{2} \mathrm{M}, 2-\mathrm{M}, \mathrm{CS}, \mathrm{HT}$ are $(0,0),(5,0)$, $(2,0)$ and $(5,0)$ respectively.

Our graphical method for variable selection is applied after running the M$\mathrm{CMC}$ and hence is computationally efficient. The selected variables can be ascertained by looking only at one $b_{i}$-v.s. $-H_{b_{i}}$ plot, which typically takes much less 
time to obtain than running a full MCMC chain.
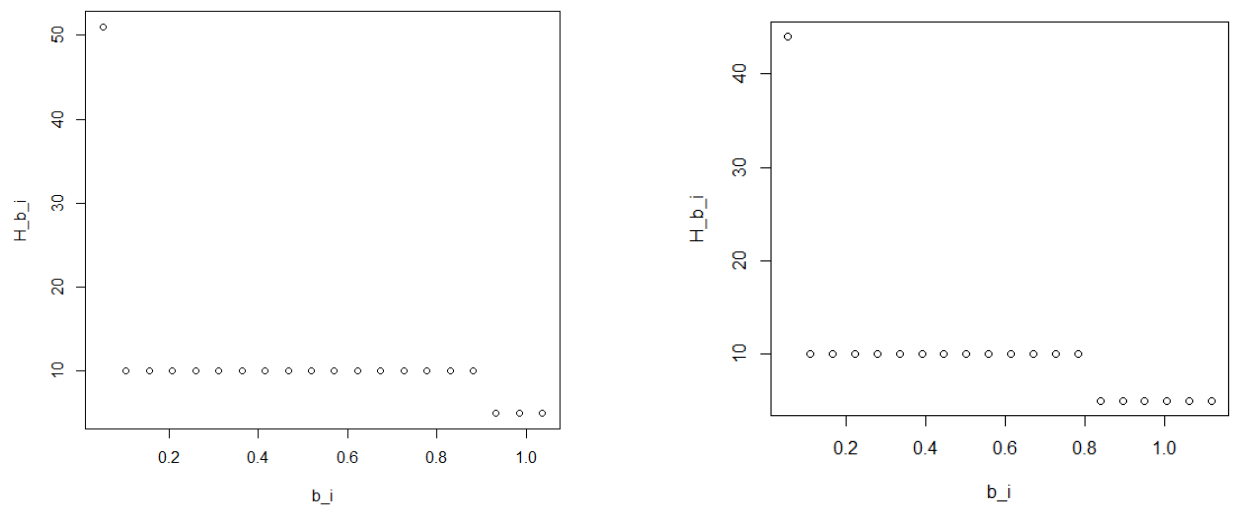

Figure 2: The $b_{i}$-v.s- $H_{b_{i}}$ plots under two settings: left: $n=50, p=300, r=$ $10, \beta_{T}=\beta_{T 1}$; right: $n=50, p=200, r=10, \beta_{T}=\beta_{T 2}$

\section{Real data application}

In this section, we explore the performance of $\mathrm{S}_{2} \mathrm{M}$ on a real data application. We consider the lymphoma dataset [25], which consists of 240 observations and 7399 features representing 4128 genes from the Lymphochip cDNA microarray. For each patient, a potentially right-censored survival time is available. The goal is to select the important genes which are predictive of the overall survival after chemotherapy. We added one unit to the survival times of all the patients, since some of the survival times in the original data set are exactly 0 . We then took the logarithm of the shifted survival times as the response in the linear regression model (1).

7399 univariate linear models using each covariate respectively are fitted and 1171 covariates whose corresponding p-values are less than 0.05 are pre-selected. This is similar to the sure independence screening [26] which is guaranteed to perform well unless the variables are highly correlated. All covariates are standardized. Among the $\left(\begin{array}{c}1171 \\ 2\end{array}\right)$ pairs of covariates, 846 pairs have correlations above 0.8 , and 236 pairs have correlations above 0.9. Out of 240 observations, 102 are right-censored. We standardized the response by the mean and standard deviation of those 138 uncensored observations only [27]. An intercept is included in the model before performing the variable selection. The MCMC was run for 30,000 
iterations with the first 6,000 as burn-in. Figures B.10 and B.11 in Appendix B contain trace plots and autocorrelation plots of the 10 top selected genes. Standard tests for checking stationarity of the Markov chain are applied, and 1107 genes out of 1171 passed the test (corresponding test p-values $>0.05$ ).

$\mathrm{HS}+\mathrm{S}_{2} \mathrm{M}$ selected 78, HS+2-M selected 74, Adaptive Lasso selected 8, and Lasso selected 108 covariates. Table 2 shows the 10 genes with the highest absolute magnitudes selected by $\mathrm{S}_{2} \mathrm{M}$, Adaptive Lasso and Lasso. The estimates of the intercept are very close to the mean of the response for all the methods. Since the estimated intercept is much higher than any estimated coefficient, the intercept was discarded before applying $\mathrm{S}_{2} \mathrm{M}$. CS (defined in Section 4) selected only one gene (gene ID: 27184) while HT was not able to distinguish any signal from noise and selected no genes.

Among 78 genes selected by $\mathrm{HS}+\mathrm{S}_{2} \mathrm{M}, 25$ of them belong to the four signature groups of genes identified in [25]. These four signature groups of genes (germinal-centre B-cell signature, major histocompatibility complex class II signature, lymph-node signature, and proliferation signature) are believed to be biologically related to the disease. The numbers are 19 out of 108 for Lasso and 4 out of 8 for adaptive Lasso. We also observed there is a significant overlap between the selected genes from all the three methods. In particular, all genes selected by adaptive Lasso are also selected by Lasso, while 7 out of 8 genes selected by adaptive Lasso are selected by $\mathrm{HS}+\mathrm{S}_{2} \mathrm{M}$. In addition, 53 genes are common between 78 selected by $\mathrm{HS}+\mathrm{S}_{2} \mathrm{M}$ and 108 selected by Lasso. The qq-plot of the residuals, and the residuals-v.s.-fitted plot (for the horseshoe implementation) are also provided on Figure 3 to demonstrate the validity of the linear model.

Table 2: Top 10 genes (with largest absolute value of coefficients)

\begin{tabular}{cc}
\hline & Unique ID \\
\hline HS+S ${ }_{2} \mathrm{M}$ & $27184,29136,29108,23942,16357,27321,20170,31634,16828,31983$ \\
\hline Adaptive Lasso & $28641,27184,29108,17154,34534,20170,32679,31981$ \\
\hline Lasso & $29108,20170,27184,34534,28641,16600,29657,17154,15930,32679$ \\
\hline
\end{tabular}

\section{Discussion}

In this article, we developed a simple but useful method for doing variable selection using shrinkage priors by post-processing posterior samples of the regression coefficients. Our method is essentially applicable to any prior and is 

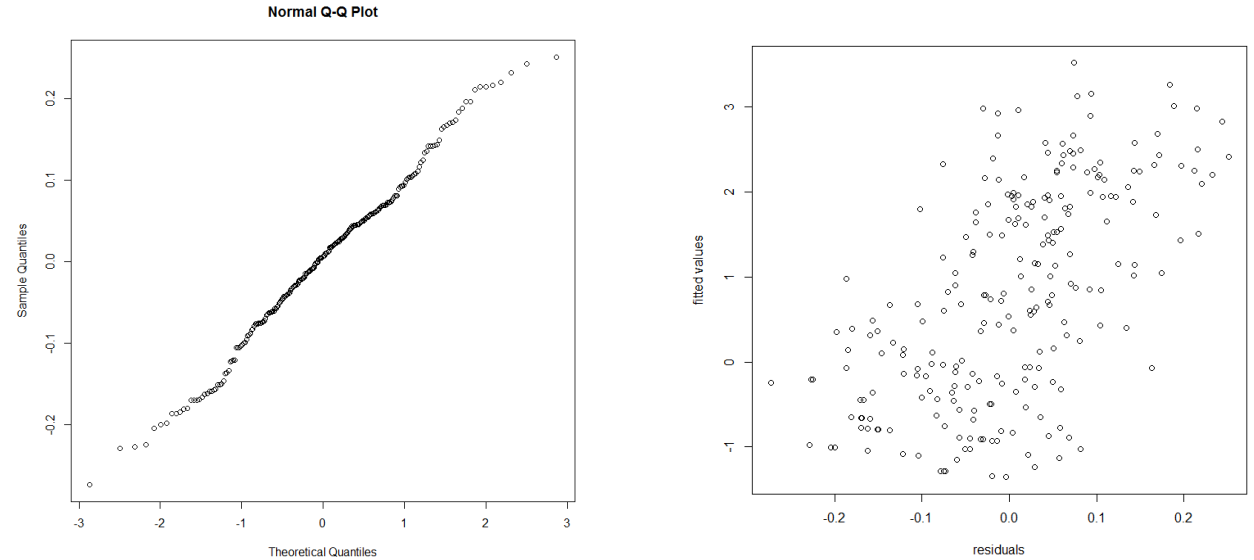

Figure 3: The qq-plot and the residual-v.s.-fitted plot for the horseshoe implementation

based on only one tuning parameter. We observe excellent performances of our method in terms of computational efficiency and capability of dealing with correlated covariates. The only tuning parameter associated with this method plays a key role to minimize the chance of masking while keeping the chance of swamping low as well. Although our current proposal for the tuning parameter works well in most situations we tried, we would like to explore a theoretically rigorous way to choose this tuning parameter in future. In presence of high-correlation, one may need more sensitivity analyses and expert domain knowledge to obtain a scientifically meaningful pre-screening.

The theoretical results are restricted to priors with only global shrinkage parameters. Although we have provided several numerical analysis to better understand the shrinkage properties for the horseshoe prior when the covariates are highly correlated, we aim to study reverse-shrinkage more rigorously for the general class of global-local priors (2) in future.

\section{Appendix A. Proof of Theorem 3.1 and supporting results}

Lemma Appendix A.1. Define function $h$ as

$$
h(x)=\int_{\beta} N\left(x ; \beta, \sigma^{2}\left(X^{\prime} X\right)^{-1}\right) \pi(\beta) d \beta .
$$


With normal priors on $\beta$ and $X^{\prime} X$ as in Theorem 1 , the function $h$ can be written as:

$$
h\left(x_{1}, x_{2}\right)=C \sigma^{-2} \sqrt{\frac{\kappa^{2}}{1-(1-\kappa)^{2} \rho^{2}}} \exp \left\{\frac{1}{2 \sigma^{2}}\left(f_{1} x_{1}^{2}+f_{2} x_{2}^{2}+2 f_{3} x_{1} x_{2}\right)\right\},
$$

where $\kappa=1 /\left(1+\tau^{2}\right)$, $C$ is a constant independent from $\sigma, \tau, \rho, x_{1}, x_{2}$, and

$$
f_{1}(\kappa ; \rho)=f_{2}(\kappa ; \rho)=\frac{\left(\rho^{2}-1-\rho^{2} \kappa\right) \kappa}{1-(1-\kappa)^{2} \rho^{2}}, \quad f_{3}(\kappa ; \rho)=-\frac{\rho \kappa^{2}}{1-(1-\kappa)^{2} \rho^{2}} .
$$

Proof. Through the calculations below, $C$ represents different constant numbers from step to step. However, $C$ is always independent from $\sigma, \tau, \rho, x_{1}$ and $x_{2}$.

$$
\begin{gathered}
h\left(x_{1}, x_{2}\right)=\int_{\beta_{1}, \beta_{2}} \frac{1}{2 \pi \sigma^{2}} \exp \left\{-\frac{1}{2 \sigma^{2}}\left[\left(x_{1}-\beta_{1}\right)^{2}+\left(x_{2}-\beta_{2}\right)^{2}+2 \rho\left(x_{1}-\beta_{1}\right)\left(x_{2}-\beta_{2}\right)\right]\right\} \\
\frac{1}{\left(2 \pi \sigma^{2} \tau^{2}\right)} \exp \left\{-\frac{1}{2 \sigma^{2} \tau^{2}}\left(\beta_{1}^{2}+\beta_{2}^{2}\right)\right\} d \beta_{1} d \beta_{2} .
\end{gathered}
$$

First we try to integrate $\beta_{1}$ out, obtaining

$h\left(x_{1}, x_{2}\right)=C \int_{\beta_{2}} \frac{1}{\sigma^{4} \tau^{2}} \exp \left\{-\frac{1}{2 \sigma^{2}}\left(x_{1}^{2}+x_{2}^{2}+\beta_{2}^{2}+\frac{1}{\tau^{2}} \beta_{2}^{2}-2 x_{2} \beta_{2}+2 \rho x_{1} x_{2}-2 \rho x_{1} \beta_{2}\right)\right\} I_{1} d \beta_{2}$,

where $I_{1}=\sqrt{2 \pi \mu} \sigma \exp \left\{\frac{1}{2 \sigma^{2}} B_{1}\right\}$, with $B_{1}^{2}=A_{1}^{-1}\left(\rho x_{2}+x_{1}-\rho \beta_{2}\right)^{2}, A_{1}^{2}=\frac{\tau^{2}+1}{\tau^{2}}$ and $\mu=\frac{\tau^{2}}{1+\tau^{2}}$.

Substitute $I_{1}$ and $B_{1}^{2}$ back to (A.4), and continue to integrate (A.4) with respect to $\beta_{2}$, obtaining

$$
h\left(x_{1}, x_{2}\right)=C \sigma^{-3} \tau^{-2} \exp \left\{-\frac{1}{2 \sigma^{2}}\left(x_{1}^{2}+x_{2}^{2}+2 \rho x_{1} x_{2}\right)\right\} \exp \left\{\frac{u}{2 \sigma^{2}}\left(\rho x_{2}+x_{1}\right)^{2}\right\} I_{2},
$$

where $I_{2}=\sqrt{2 \pi} \sigma \exp \left\{\frac{1}{2 \sigma^{2}} B_{2}^{2}\right\} \sqrt{\frac{\mu}{1-\mu^{2} \rho^{2}}}$, with $B_{2}^{2}=A_{2}^{-2}\left[\mu \rho\left(\rho x_{2}+x_{1}\right)-\left(\rho x_{1}+\right.\right.$ $\left.\left.x_{2}\right)\right]^{2}$ and $A_{2}^{2}=\frac{1}{\mu}-\mu \rho^{2}=\frac{1-\mu^{2} \rho^{2}}{\mu}$.

Substituting $I_{2}$ with $B_{2}^{2}$ back to (A.5), and letting $\kappa=1-\mu=\frac{1}{1+\tau^{2}}$, (A.2) can be obtained.

Lemma Appendix A.2. Suppose $\left|\hat{\beta}_{M L E, 1}\right|=A\left|\hat{\beta}_{M L E, 2}\right|, \hat{\beta}_{M L E}=\left(\hat{\beta}_{M L E, 1}, \hat{\beta}_{M L E, 2}\right)$, 
then the normal estimators can be written as functions of the MLEs as:

$$
\begin{aligned}
& \hat{\beta}_{N, 1}=\left[1-\frac{1}{1-\rho^{2}}\left(R_{1}\left(\hat{\beta}_{M L E}\right)-\frac{\rho R_{2}\left(\hat{\beta}_{M L E}\right)}{A}\right)\right] \hat{\beta}_{M L E, 1}, \\
& \hat{\beta}_{N, 2}=\left[1-\frac{1}{1-\rho^{2}}\left(R_{1}\left(\hat{\beta}_{M L E}\right)-\rho R_{2}\left(\hat{\beta}_{M L E}\right) A\right)\right] \hat{\beta}_{M L E, 2}
\end{aligned}
$$

where $R_{1}\left(\hat{\beta}_{M L E}\right)=-\frac{1}{A}\left(A f_{1}(\kappa)+f_{3}(\kappa)\right)$ and $R_{2}\left(\hat{\beta}_{M L E}\right)=-\left(f_{2}(\kappa)+A f_{3}(\kappa)\right)$.

Proof. Continuing the Lemma Appendix A.1, the derivatives of the function $h\left(x_{1}, x_{2}\right)$ with respective to $x_{i}$ is

$$
\frac{\partial}{\partial x_{i}} h\left(x_{1}, x_{2}\right)=C \sigma^{-2}\left(f_{i} x_{i}+f_{3} x_{3-i}\right) h\left(x_{1}, x_{2}\right), \quad i=1,2 .
$$

where $C$ here denotes the constant before the exponential in the function $h$. Define

$$
R_{i}^{*}\left(x_{1}, x_{2}\right)=-\frac{1}{x_{i}} \frac{\frac{\partial}{\partial x_{i}} h}{h}=-\frac{1}{\sigma^{2} x_{i}}\left(f_{i} x_{i}+f_{3} x_{3-i}\right), \quad i=1,2 .
$$

Considering $\left|\hat{\beta}_{M L E, 1}\right|=A\left|\hat{\beta}_{M L E, 2}\right|$, both $R_{1}^{*}\left(\hat{\beta}_{M L E}\right)$ and $R_{2}^{*}\left(\hat{\beta}_{M L E}\right)$ can be written as functions of $A$ as in Lemma. Using the result of the Proposition 1 in [15], where

$S(\hat{\beta})=\sigma^{2}\left(X^{\prime} X\right)^{-1}\left[R_{1}^{*}\left(\hat{\beta}_{M L E}\right) 0 ; 0 R_{2}^{*}\left(\hat{\beta}_{M L E}\right)\right]=\left(X^{\prime} X\right)^{-1}\left[R_{1}\left(\hat{\beta}_{M L E}\right), R_{2}\left(\hat{\beta}_{M L E}\right)\right]$,

in this case, (A.6) can be obtained.

Lemma Appendix A.3. Define

$$
S_{1}=\frac{\left(R_{1}-\frac{\rho R_{2}}{A}\right)}{1-\rho^{2}}, S_{2}=\frac{\left(R_{2}-\rho R_{1} A\right)}{1-\rho^{2}},
$$

then $-1<f_{1}(\kappa ; \rho)=f_{2}(\kappa ; \rho)<f_{3}(\kappa ; \rho)<0,0<S_{1}<1, S_{2}<1$, for any $0<\rho<1, A>1$, and $\tau>0$.

Proof. $f_{1}=f_{2}$ and $f_{3}<0$ can be directly obtained from the definitions (A.3).

$f_{1}(\kappa ; \rho)>-1 \Leftrightarrow \frac{\left(\rho^{2}-1-\rho^{2} \kappa\right) \kappa}{1-(1-\kappa)^{2} \rho^{2}}>-1 \Leftrightarrow \rho^{2} \kappa-\kappa-\rho^{2} \kappa^{2}>\left(\kappa^{2}+1-2 \kappa\right) \rho^{2}-1$ (A.9)

For the last inequality in (A.9), $L H S-R H S=2 \rho^{2} \kappa(1-\kappa)+\left(1-\rho^{2}\right)(1-\kappa)>0$. 
Thus $f_{1}>-1$.

$$
f_{3}>f_{1} \Leftrightarrow\left(\rho^{2}-1-\rho^{2} \kappa\right) \kappa<-\rho \kappa^{2} .
$$

For the inequality in (A.10), LHS - RHS $=(1-\rho)\left(\rho \kappa^{2}-\kappa-\rho\right)<0$, this leads to $f_{3}>f_{1}$.

Since $f_{1}, f_{2}$ and $f_{3}$ are always negative, $R_{1}$ and $R_{2}$ are always positive.

$$
S_{1}>0 \Leftrightarrow R_{1}>\frac{\rho R_{2}}{A} \Leftrightarrow \frac{R_{1}}{R_{2}}>\frac{\rho}{A} .
$$

We proved $\frac{R_{1}}{R_{2}}>\frac{A+\rho}{A+\rho A^{2}}$. Considering the fact $\frac{A+\rho}{A+\rho A^{2}}>\frac{\rho}{A}$, the last inequality of (A.11) is true. Thus $S_{1}>0$.

We prefer to use $\mu=1-\kappa=\tau^{2} /\left[1+\tau^{2}\right]$ to prove $S_{1}<1$ :

$$
S_{1}<1 \Leftrightarrow R_{1}-\frac{\rho R_{2}}{A}<1-\rho^{2} \Leftrightarrow A R_{1}-\rho R_{2}<A-A \rho^{2} .
$$

For the last inequality in (A.12), we can finally obtain $L H S-R H S=(1-$ $\left.\rho^{2}\right)\left(\rho \mu^{2}-\rho \mu+A \rho^{2} \mu^{2}-A \mu\right)$. Since both $\rho \mu(\mu-1)$ and $A \mu(\rho \mu-1)$ are negative, LHS - RHS is less than 0 .

$$
S_{2}<1 \Leftrightarrow R_{2}-\rho R_{1} A<1-\rho^{2} .
$$

For the inequality in (A.13), $L H S-R H S=\left(1-\rho^{2}\right)\left[\left(A \rho \mu^{2}-A \rho \mu\right)+\left(\rho^{2} \mu^{2}-\mu\right)\right]$. Since both $\left(A \rho \mu^{2}-A \rho \mu\right)$ and $\left(\rho^{2} \mu^{2}-\mu\right)$ are negative, thus $L H S$ is indeed less than $R H S$.

Now we are ready to give the proof of Theorem 3.1.

Proof. Case 1: the Theorem is true when $S_{2}<0$. That is because $S_{1}$ is always between 0 and 1 , causing $\left|\hat{\beta}_{N, 1}\right|$ will always be less than $\left|\hat{\beta}_{M L E, 1}\right|$. But $\left|\hat{\beta}_{N, 2}\right|$ will be greater than $\left|\hat{\beta}_{M L E, 2}\right|$ since $\left(1-S_{2}\right)$ must be greater than 1 in this case.

Case 2: when both $S_{1}$ and $S_{2}$ are between 0 and 1, we have

$$
(3) \Leftrightarrow \frac{S_{1}}{S_{2}}>1 \Leftrightarrow \frac{R_{1}-\frac{\rho R_{2}}{A}}{R_{2}-\rho R_{1} A}>1 \Leftrightarrow A R_{1}-\rho R_{2}>A R_{2}-\rho R_{1} A^{2} .
$$

For the last inequality in (A.14), substituting $R_{1}$ and $R_{2}$ derived from Lemma Appendix A.2, we can obtain $L H S-R H S=\rho\left(1-\rho^{2}\right)\left(A^{2}-1\right)(1-\kappa)>0$.

Note this verifies $\frac{R_{1}}{R_{2}}>\frac{A+\rho}{A+\rho A^{2}}$, which is needed for proving $S_{1}>0$ earlier. 
Lemma Appendix A.4. The function $h$ is defined as (A.1), under the model (1) with the horseshoe prior on $\beta$, then

$$
h\left(x_{1}, x_{2}\right)=C \int_{\kappa_{1}, \kappa_{2}} F\left(\kappa_{1}, \kappa_{2} ; \rho\right) E\left(\kappa_{1}, \kappa_{2} ; \rho, x_{1}, x_{2}\right) d \kappa_{1} d \kappa_{2}
$$

where $\kappa_{i}=1 /\left[1+\tau^{2} \lambda_{i}^{2}\right], i=1,2, C$ is a constant independent from $\left(x_{1}, x_{2}, \rho, \lambda_{1}, \lambda_{2}\right)$, and

$$
\begin{aligned}
F\left(\kappa_{1}, \kappa_{2} ; \rho\right)= & {\left[1-\left(1-\kappa_{1}\right)\left(1-\kappa_{2}\right) \rho^{2}\right]^{-\frac{1}{2}}\left[1-\left(1-\tau^{2}\right) \kappa_{1}\right]^{-1} } \\
& \times\left[1-\left(1-\tau^{2}\right) \kappa_{2}\right]^{-1}\left(1-\kappa_{1}\right)^{-\frac{1}{2}}\left(1-\kappa_{2}\right)^{-\frac{1}{2}}, \\
E\left(\kappa_{1}, \kappa_{2} ; \rho, x_{1}, x_{2}\right)= & \exp \left\{\frac{1}{2 \sigma^{2}}\left(f_{1} x_{1}^{2}+f_{2} x_{2}^{2}+2 f_{3} x_{1} x_{2}\right)\right\}
\end{aligned}
$$

with

$f_{i}\left(\kappa_{1}, \kappa_{2} ; \rho\right)=\left[\left(\rho^{2}-1-\rho^{2} \kappa_{3-i}\right) \kappa_{i}\right]\left[1-\left(1-\kappa_{1}\right)\left(1-\kappa_{2}\right) \rho^{2}\right]^{-1}, \quad i=1,2 ;$

$f_{3}\left(\kappa_{1}, \kappa_{2} ; \rho\right)=-\rho \kappa_{1} \kappa_{2}\left[1-\left(1-\kappa_{1}\right)\left(1-\kappa_{2}\right) \rho^{2}\right]^{-1}$.

It follows that the horseshoe estimator can be represented as the right hand side of (A.6), where $R_{i}$ is

$R_{i}\left(x_{1}, x_{2}\right)=-\frac{1}{x_{i}} \frac{\int_{\kappa_{1}, \kappa_{2}}\left[f_{i}\left(\kappa_{1}, \kappa_{2}\right) x_{i}+f_{3}\left(\kappa_{1}, \kappa_{2}\right) x_{3-i}\right] F\left(\kappa_{1}, \kappa_{2}\right) E\left(\kappa_{1}, \kappa_{2}\right) d \kappa_{1} d \kappa_{2}}{\int_{\kappa_{1} \kappa_{2}} F\left(\kappa_{1}, \kappa_{2}\right) E\left(\kappa_{1}, \kappa_{2}\right) d \kappa_{1} d \kappa_{2}}$,

where $i=1,2$.

Proof. With the horseshoe prior being applied, the $\pi(\beta)$ in (A.2) becomes

$$
\begin{aligned}
\pi(\beta) & =\int_{\lambda} \pi(\beta \mid \lambda) \pi(\lambda) d \lambda \\
& =\int_{\lambda_{1}, \lambda_{2}} N\left(\beta_{1} ; 0, \sigma^{2} \tau^{2} \lambda_{1}^{2}\right) N\left(\beta_{2} ; 0, \sigma^{2} \tau^{2} \lambda_{2}^{2}\right) \pi\left(\lambda_{1}\right) \pi\left(\lambda_{2}\right) d \lambda_{1} d \lambda_{2}
\end{aligned}
$$

The function $h$ now is an integral with respect to $\left(\beta_{1}, \beta_{2}, \lambda_{1}, \lambda_{2}\right)$. The trick here is to integrate $\beta_{1}, \beta_{2}$ out, then $h$ will be an integral with respect to $\lambda_{1}, \lambda_{2}$ only:

$$
h(x)=\int_{\lambda} \pi(\lambda)\left\{\int_{\beta} N\left(x ; \beta, \sigma^{2}\left(X^{\prime} X\right)^{-1}\right) \pi(\beta \mid \lambda) d \beta\right\} d \lambda .
$$

Note $\pi(\beta \mid \lambda)$ is a two-dimension normal distribution, with $\kappa_{1}$ and $\kappa_{2}$ defined in this Lemma, we can easily integrate $\beta_{1}, \beta_{2}$ out through the similar approach as 
we did in the proof of Lemma Appendix A.1, and the integral with respect to $\beta$ inside the braces above is

$$
\int_{\beta}=C^{\prime} \frac{\sqrt{\kappa_{1} \kappa_{2}}}{\sqrt{1-\left(1-\kappa_{1}\right)\left(1-\kappa_{2}\right) \rho^{2}}} E\left(\kappa_{1}, \kappa_{2} ; \rho, x_{1}, x_{2}\right),
$$

where $C^{\prime}$ is a constant independent from $\left(x_{1}, x_{2}, \rho, \lambda_{1}, \lambda_{2}\right)$.

The prior on $\lambda_{i}$ is $\pi\left(\lambda_{i}\right)=2 /\left[\pi\left(1+\lambda_{i}^{2}\right)\right]$ then the prior on $\kappa_{i}$ is followed as:

$$
\pi\left(\kappa_{i}\right)=\frac{\tau}{\pi} \frac{1}{1-\left(1-\tau^{2}\right) \kappa_{i}}\left(1-\kappa_{i}\right)^{-\frac{1}{2}} \kappa_{i}^{-\frac{1}{2}}
$$

Substituting $\int_{\beta}$ and $\pi\left(\kappa_{i}\right)$ back to (A.17), (A.15) is obtained. Considering

$$
\frac{\partial}{\partial x_{i}} h\left(x_{1}, x_{2}\right)=C \sigma^{-2} \int_{\kappa_{1}, \kappa_{2}}\left(f_{i} x_{i}+f_{3} x_{3-i}\right) F\left(\kappa_{1}, \kappa_{2}\right) E\left(\kappa_{1}, \kappa_{2}\right) d \kappa_{1} d \kappa_{2}
$$

and defining $R_{i}=\sigma^{2} R_{i}^{*}-\left(\frac{\partial}{\partial x_{i}} h\right) /\left(x_{i} h\right)$, use the result of Proposition 1 in [15] again, (A.16) is obtained.

\section{Appendix B. MCMC diagnostics}

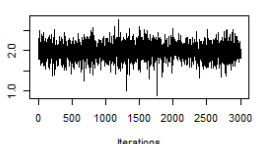

therations
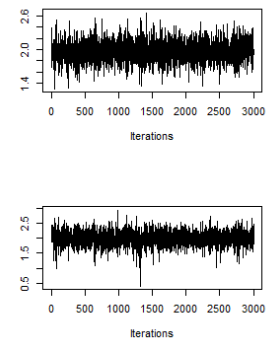
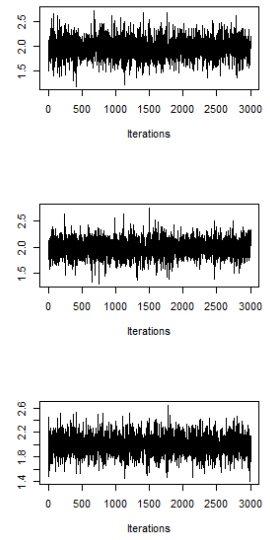

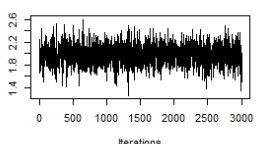

Herations
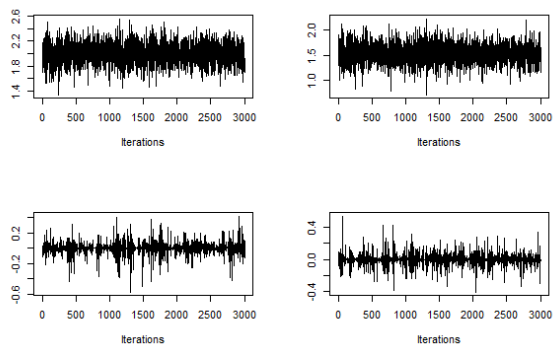

Figure B.4: The trace plots (in a typical simulation using the horseshoe prior) of 10 signals and 2 randomly selected noise covariates 

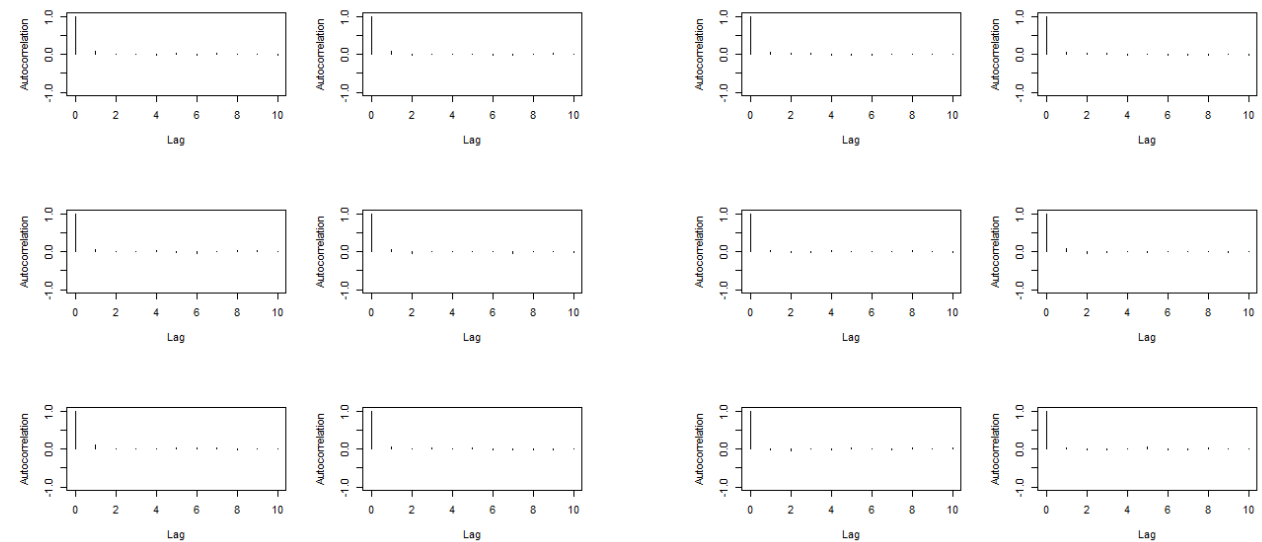

Figure B.5: The autocorrelation plots (in a typical simulation using the horseshoe prior) of 10 signals and 2 randomly selected noise covariates

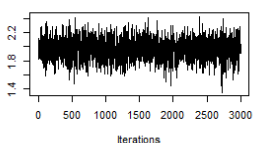

Hterations
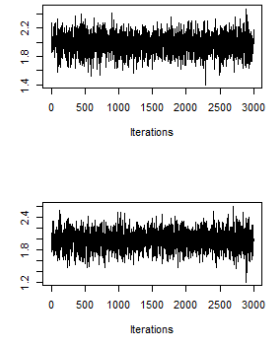
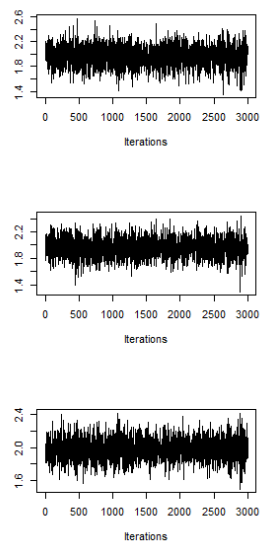
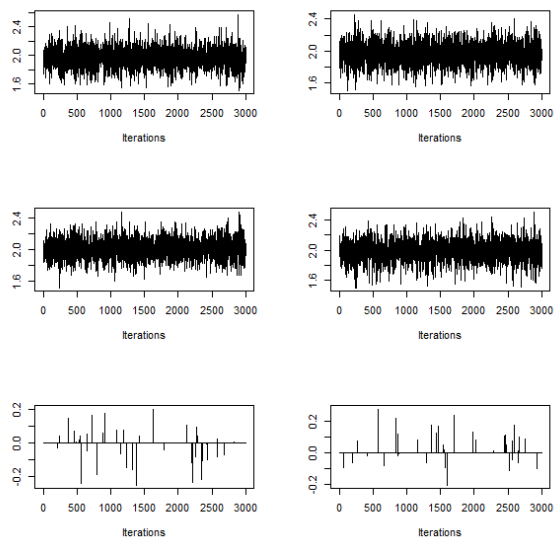

Figure B.6: The trace plots (in a typical simulation using the spike and slab prior) of 10 signals and 2 randomly selected noise covariates 

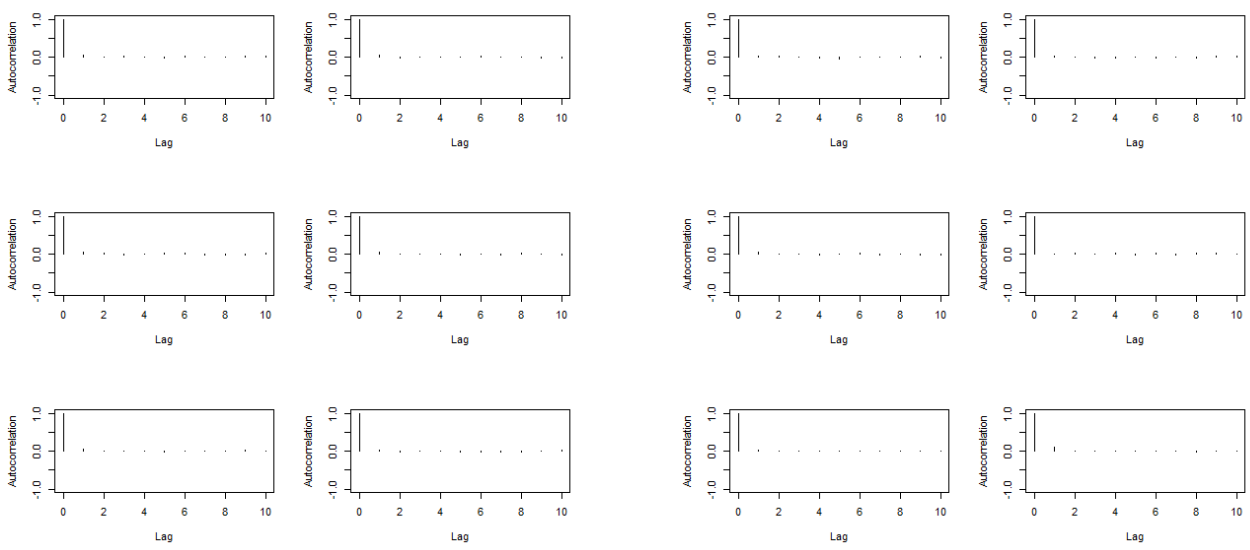

Figure B.7: The autocorrelation plots (in a typical simulation using the spike and slab prior) of 10 signals and 2 randomly selected noise covariates
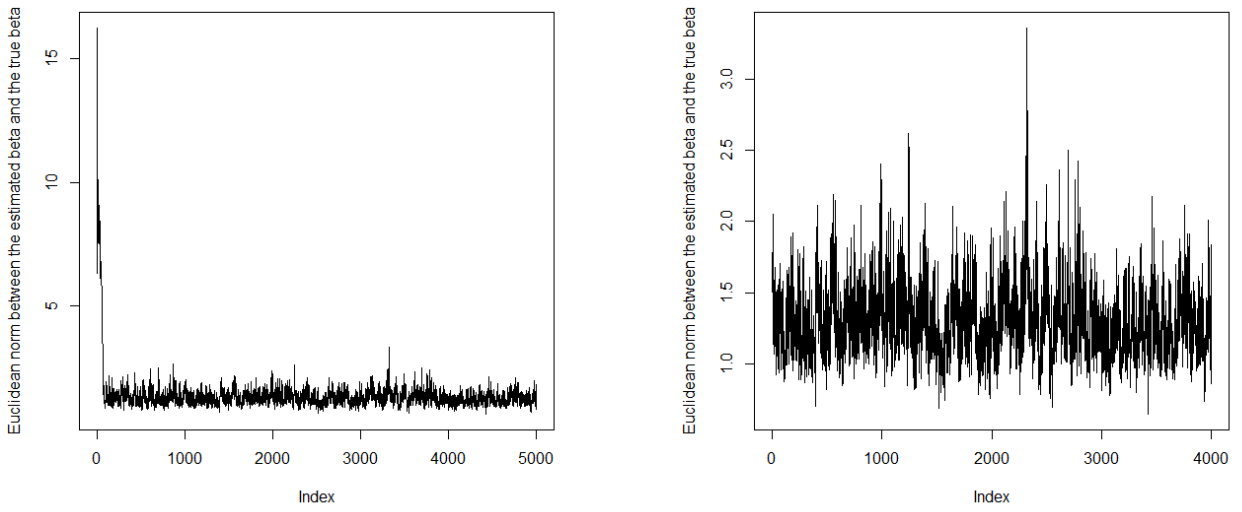

Figure B.8: Euclidean norm between the estimated $\beta$ at each iteration and the true $\beta$ in a typical simulation using the horseshoe prior. Left: beginning from the 1st iteration; right: beginning from the 1001th iteration 

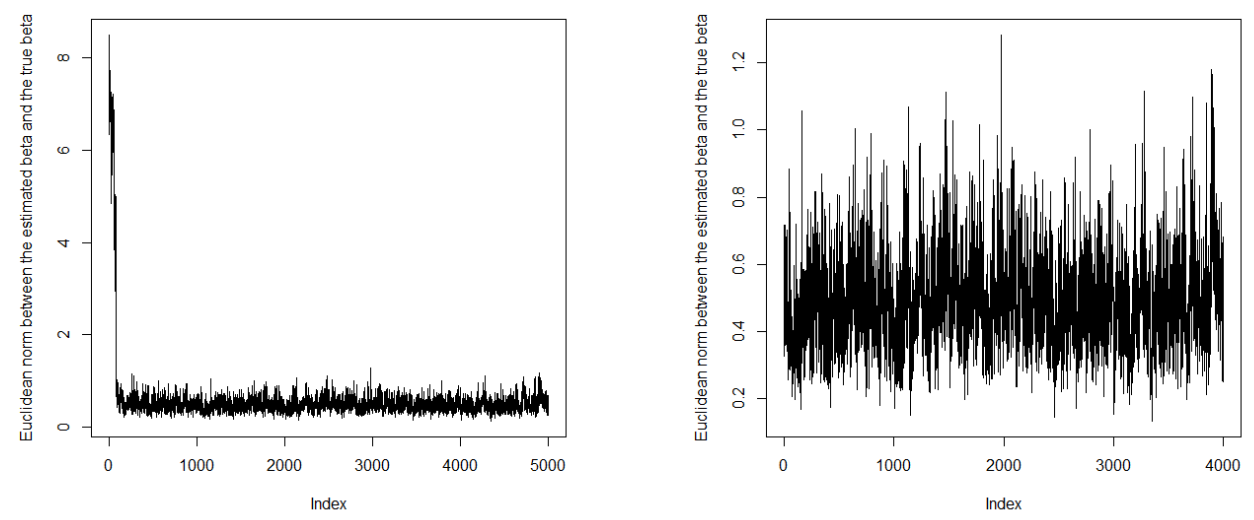

Figure B.9: Euclidean norm between the estimated $\beta$ at each iteration and the true $\beta$ in a typical simulation using the spike and slab prior. Left: beginning from the 1st iteration; right: beginning from the 1001th iteration
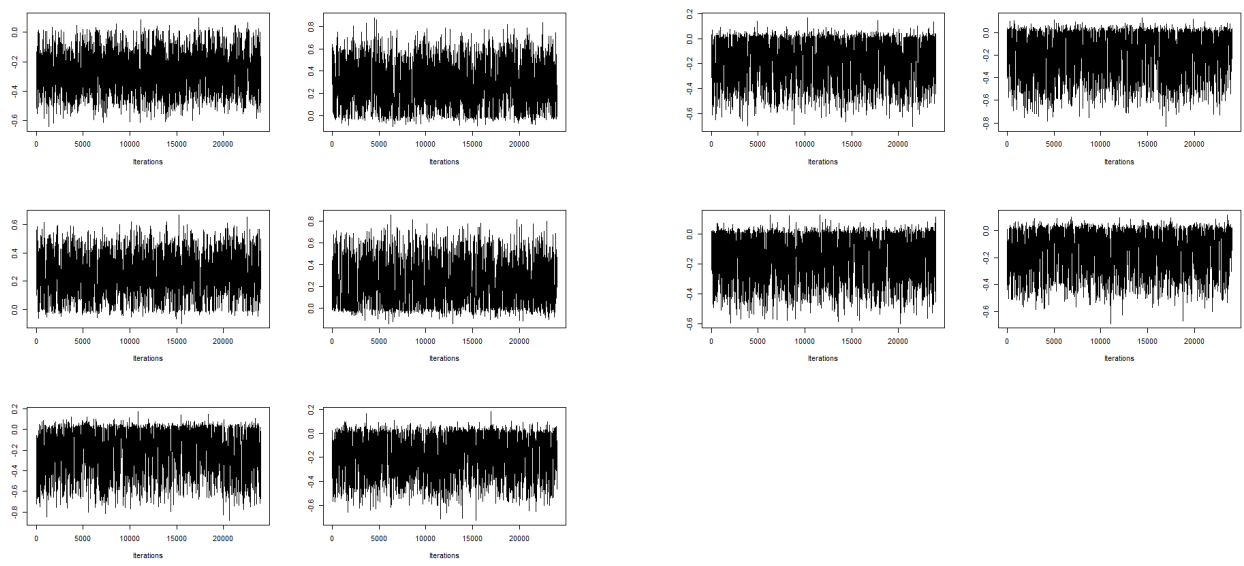

Figure B.10: The trace plots corresponding to the 10 genes from $\mathrm{HS}+\mathrm{S}_{2} \mathrm{M}$ on Table 2 

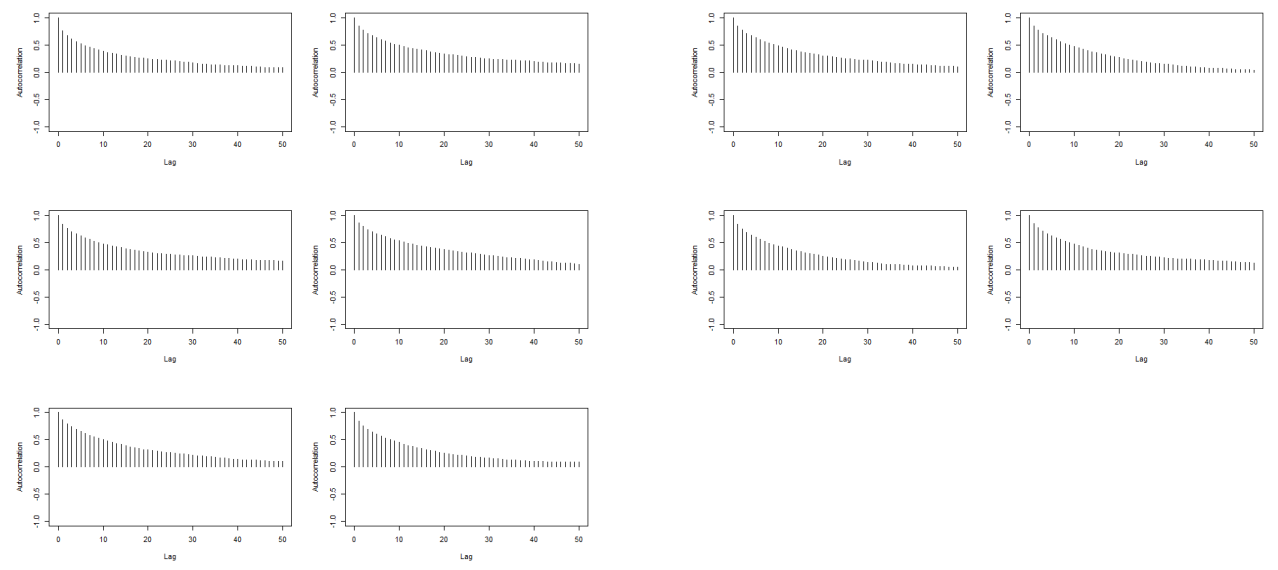

Figure B.11: The autocorrelation plots of corresponding to the 10 genes from $\mathrm{HS}+\mathrm{S}_{2} \mathrm{M}$ on Table 2 
[1] J. Fan, J. Lv, A selective overview of variable selection in high dimensional feature space, Statistica Sinica 20 (1) (2010) 101.

[2] T. J. Mitchell, J. J. Beauchamp, Bayesian variable selection in linear regression, Journal of the American Statistical Association 83 (404) (1988) 1023-1032.

[3] E. George, R. McCulloch, Variable selection via Gibbs sampling, Journal of the American Statistical Association 88 (423) (1993) 881-889.

[4] E. I. George, R. E. McCulloch, Approaches for Bayesian variable selection, Statistica sinica 7 (2) (1997) 339-373.

[5] V. Johnson, D. Rossell, On the use of non-local prior densities in Bayesian hypothesis tests, Journal of the Royal Statistical Society: Series B (Statistical Methodology) 72 (2) (2010) 143-170.

[6] J. Berger, L. Pericchi, The intrinsic Bayes factor for model selection and prediction, Journal of the American Statistical Association 91 (433) (1996) 109-122.

[7] A. O'Hagan, Fractional bayes factors for model comparison, Journal of the Royal Statistical Society. Series B (Methodological) (1995) 99-138.

[8] F. Liang, R. Paulo, G. Molina, M. Clyde, J. Berger, Mixtures of g priors for Bayesian variable selection, Journal of the American Statistical Association 103 (481).

[9] M. A. Clyde, Bayesian model averaging and model search strategies, Bayesian statistics 6 (1999) 157-185.

[10] M. A. Clyde, E. I. George, Empirical bayes estimation in wavelet nonparametric regression, in: Bayesian inference in wavelet-based models, Springer, 1999, pp. 309-322.

[11] M. M. Barbieri, J. O. Berger, Optimal predictive model selection, Annals of Statistics (2004) 870-897.

[12] J. Ghosh, A. E. Ghattas, Bayesian variable selection under collinearity.

[13] T. Park, G. Casella, The Bayesian lasso, Journal of the American Statistical Association 103 (482) (2008) 681-686. 
[14] M. Tipping, Sparse Bayesian learning and the relevance vector machine, The Journal of Machine Learning Research 1 (2001) 211-244.

[15] J. Griffin, P. Brown, Inference with normal-gamma prior distributions in regression problems, Bayesian Analysis 5 (1) (2010) 171-188.

[16] C. Carvalho, N. Polson, J. Scott, The horseshoe estimator for sparse signals, Biometrika 97 (2) (2010) 465-480.

[17] C. Carvalho, N. Polson, J. Scott, Handling sparsity via the horseshoe, in: International Conference on Artificial Intelligence and Statistics, 2009, pp. 73-80.

[18] A. Bhattacharya, D. Pati, N. Pillai, D. Dunson, Dirichlet-laplace priors for optimal shrinkage, Journal of the American Statistical Association, to appear.

[19] N. Polson, J. Scott, Shrink globally, act locally: sparse Bayesian regularization and prediction, Bayesian Statistics 9 (2010) 501-538.

[20] P. R. Hahn, C. M. Carvalho, Decoupling shrinkage and selection in bayesian linear models: a posterior summary perspective, Journal of the American Statistical Association (just-accepted) (2015) 00-00.

[21] H. Bondell, B. Reich, Consistent high-dimensional Bayesian variable selection via penalized credible regions, Journal of the American Statistical Association 107 (500) (2012) 1610-1624.

[22] A. Vehtari, J. Lampinen, Bayesian input variable selection using posterior probabilities and expected utilities, Report B31.

[23] A. Bhattacharya, D. Pati, N. S. Pillai, D. B. Dunson, Bayesian shrinkage, arXiv preprint arXiv:1212.6088.

[24] H. Zou, The adaptive lasso and its oracle properties, Journal of the American statistical association 101 (476) (2006) 1418-1429.

[25] A. Rosenwald, G. Wright, W. C. Chan, J. M. Connors, E. Campo, R. I. Fisher, R. D. Gascoyne, H. K. Muller-Hermelink, E. B. Smeland, J. M. Giltnane, et al., The use of molecular profiling to predict survival after chemotherapy for diffuse large-b-cell lymphoma, New England Journal of Medicine 346 (25) (2002) 1937-1947. 
[26] J. Fan, J. Lv, Sure independence screening for ultrahigh dimensional feature space, Journal of the Royal Statistical Society: Series B (Statistical Methodology) 70 (5) (2008) 849-911.

[27] A. Bhattacharya, D. Dunson, Sparse Bayesian infinite factor models, Biometrika 98 (2) (2011) 291-306. 